\title{
Percutaneous vertebroplasty for Kummell's disease: Short-term analysis of therapeutic effects and outcome predictors
}

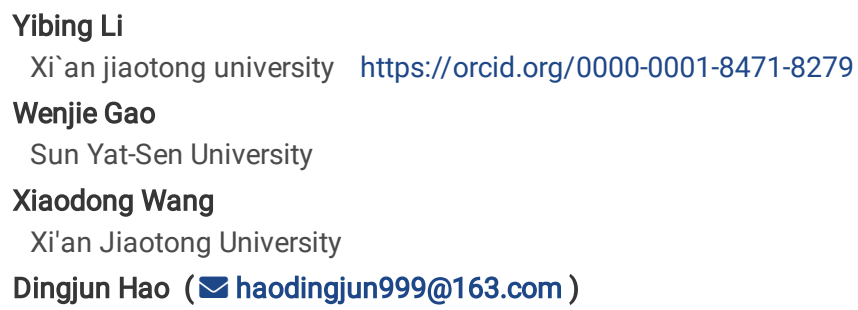




\section{Abstract}

Background: To retrospectively investigate the short-term therapeutic effect of percutaneous vertebroplasty (PVP) for Kummell's disease using a variable angle needle and to analyze the possible outcome predictors.

Methods: Twenty-seven patients were included in this study. Medical records were evaluated. The following factors were analyzed on imaging data: location of lesion, the percentage of bone marrow edema, patterns of cement spreading, and post-procedural kyphosis correction. Visual analogue score (VAS) was used to analyze the clinical effect, and the improvement rate was calculated. Statistical analyses were conducted to evaluate the relationship between those factors and therapeutic outcomes using Fisher's exact test, Chi-squared test, and Student's unpaired t-test.

Results: The effectiveness rate was $70.4 \%$ at 1 week, $74.1 \%$ after 1 month, and $63.0 \%$ after 3 months. Six months and one year after procedures, the effective rate remained steady at $70.4 \%$. Post-procedural kyphosis correction $\geq 8^{\circ}$ was a risk factor for inferior pain relief. An area of edema over $40 \%$ was a prognostic factor for superior pain relief at the first follow-up point. Cement morphology was a positive predictor for better pain relief at 1 month followup.

Conclusions: Compared to the therapeutic effects of PVP on fresh osteoporotic vertebral compression fractures (OVCF), percutaneous vertebroplasty for Kummell's disease was effective in only $63 \%$ to $74.1 \%$ of the patients at different follow-up points. Overemphasized kyphotic correction (over 8 degrees) was associated with poor outcomes. Areas of edema over $40 \%$ and cement morphology of mixed type were positive prognostic factors in the early stage.

\section{Background}

With an aging global population, osteoporotic vertebral compression fractures (OVCFs) have become increasingly common around the world. Because of the negative bone metabolism, Kummell's disease presents as one complication of OVCF, but the exact pathogenesis is still unknown. Avascular necrosis, capillary fat embolism and micro-instability might all contribute to its occurrence [1-3]. Various terms have been used to describe this disease: avascular necrosis after OVCF; delayed vertebral collapse; OVCF nonunion and post-traumatic vertebral osteonecrosis. The actual incidence varies from 7-37\% among the elderly population with OVCFs [2,3]. An osteoporotic burst or compression fracture is the initial factor, followed by dynamic instability. Cord compression with iconic intravertebral cleft (IVC) is the ultimate result in some severe cases. Percutaneous vertebroplasty (PVP) has emerged as an effective technique for treatment of painful OVCF because of its instant stabilization and pain relief effect $[4,7-9,19]$. Motion within the IVC is a cause of persistent pain. Excellent pain relief is achievable if the IVC is filled with cement [1,3,4]. However, the clinical results of PVP on Kummell's disease have not been as impressive as expected [3-5]. Patients with Kummell's disease might react discrepantly to cement augmentation. The purpose of this retrospective study was to evaluate the short-term therapeutic effects of PVP on Kummell's disease and to analyze possible risk factors for negative clinical effects.

\section{Methods}

\section{Patient selection}

The approval of the institutional review board and patient approval were not necessary for this study due to its retrospective nature. Patients' consents for PVPs were achieved preoperatively.

Forty-three consecutive patients were enrolled in the study. Criteria for Kummell's disease were (1) IVCs with an accumulation of gas or liquid visible in the sagittal and coronal reconstructive computed tomographic (CT) scans and/or sagittal magnetic resonance images (MRI) (2) symptoms or signs compatible with a focal lesion, such as tenderness around the lesion and aggravating back pain during flexion and extension; (3) typical symptom evolutions from an asymptomatic phase to a deformity phase. All operations were performed by the corresponding authors and the first author. Four to six weeks of conservative treatments including brace fixation and medications were administered preoperatively.

Exclusion criteria were as follows: (1) patients with comorbidities such as stroke, dementia, or vital system failure, which made intensive medical therapies necessary;(2) new-onset OVCFs in the follow-up period; (3) Kummell's disease with neurological deficit, which made decompression necessary; (4) previous spinal open surgery or vertebroplasty. (5) Follow-up duration of less than 12 months.

Ten patients with co-existing acute compression fractures in another vertebra were excluded to simplify the evaluation of post-procedural pain relief. Four patients were lost to the post-procedural follow-up. Two patients were excluded for comorbidities. This study ultimately included 27 patients with a mean age of 70.9 years. Demographic characteristics are shown in Table 1. 
Table 1

Demographic data and therapeutic effect on pain relief after PVP on Kummell's disease.

\begin{tabular}{|c|c|c|c|c|c|c|c|c|c|c|c|c|c|c|c|}
\hline Patients & Age(year) & Gender & BMD & BMI & Pre-V & VAS1 & VAS2 & VAS3 & VAS4 & VAS5 & KC & $\mathrm{PE}$ & $\mathrm{CV}(\mathrm{ml})$ & LFV & $\mathrm{CM}$ \\
\hline 1 & 67 & 1 & -2.9 & 23.7 & 7 & 2 & 3 & 3 & 4 & 3 & 7 & 0.56 & 4.4 & T6 & 2 \\
\hline 2 & 77 & 1 & -2.1 & 10.6 & 8 & 5 & 4 & 5 & 5 & 4 & 14 & 0.42 & 5.5 & T12 & 2 \\
\hline 3 & 65 & 1 & -3.4 & 21.3 & 7 & 3 & 4 & 4 & 3 & 2 & 7 & 0.45 & 5.3 & T8 & 2 \\
\hline 4 & 62 & 1 & -2.9 & 26.9 & 7 & 4 & 3 & 4 & 5 & 6 & 9 & 0.52 & 4.8 & T12 & 2 \\
\hline 5 & 67 & 2 & -2.6 & 25.0 & 8 & 3 & 3 & 3 & 3 & 3 & 8 & 0.61 & 6.0 & $\mathrm{~T} 11$ & 3 \\
\hline 6 & 64 & 2 & -3.0 & 29.7 & 7 & 3 & 3 & 4 & 4 & 3 & 9 & 0.39 & 5.0 & T6 & 1 \\
\hline 7 & 65 & 1 & -3.2 & 16.0 & 7 & 6 & 6 & 7 & 6 & 6 & 13 & 0.24 & 6.0 & T12 & 1 \\
\hline 8 & 66 & 2 & -2.9 & 24.0 & 9 & 3 & 3 & 5 & 3 & 3 & 8 & 0.63 & 4.0 & L1 & 3 \\
\hline 9 & 68 & 1 & -2.9 & 15.2 & 7 & 4 & 5 & 5 & 4 & 5 & 9 & 0.45 & 4.5 & L3 & 2 \\
\hline 10 & 78 & 2 & -2.3 & 15.4 & 7 & 3 & 3 & 4 & 3 & 3 & 7 & 0.62 & 5.0 & T8 & 3 \\
\hline 11 & 78 & 2 & -3.0 & 16.7 & 7 & 3 & 3 & 4 & 3 & 2 & 11 & 0.35 & 4.0 & L2 & 1 \\
\hline 12 & 78 & 2 & -2.5 & 25.7 & 8 & 5 & 5 & 5 & 4 & 6 & 10 & 0.15 & 4.6 & T12 & 1 \\
\hline 13 & 62 & 1 & -2.6 & 26.2 & 7 & 3 & 4 & 4 & 3 & 3 & 2 & 0.45 & 6.4 & L1 & 3 \\
\hline 14 & 68 & 1 & -3.1 & 8.8 & 7 & 3 & 4 & 3 & 3 & 3 & 7 & 0.40 & 5.2 & T3 & 3 \\
\hline 15 & 63 & 2 & -3.1 & 18.6 & 9 & 3 & 4 & 3 & 4 & 3 & 8 & 0.56 & 4.0 & T11 & 2 \\
\hline 16 & 64 & 2 & -3.2 & 17.3 & 6 & 5 & 5 & 4 & 3 & 5 & 14 & 0.14 & 4.0 & L2 & 1 \\
\hline 17 & 64 & 2 & -1.7 & 13.8 & 6 & 2 & 3 & 4 & 3 & 2 & 13 & 0.49 & 4.3 & L3 & 3 \\
\hline 18 & 68 & 1 & -2.5 & 27.8 & 7 & 3 & 4 & 3 & 3 & 3 & 7 & 0.35 & 4.6 & T9 & 2 \\
\hline 19 & 68 & 2 & -3.5 & 22.4 & 6 & 6 & 6 & 5 & 5 & 5 & 11 & 0.44 & 6.5 & L2 & 3 \\
\hline 20 & 70 & 2 & -2.9 & 26.3 & 8 & 5 & 5 & 5 & 5 & 6 & 8 & 0.34 & 4.5 & T10 & 2 \\
\hline 21 & 73 & 1 & -3.5 & 19.9 & 9 & 5 & 4 & 5 & 5 & 5 & 11 & 0.49 & 4.8 & T12 & 3 \\
\hline 22 & 74 & 2 & -2.7 & 12.1 & 8 & 5 & 4 & 5 & 5 & 4 & 13 & 0.38 & 4.5 & $\mathrm{~T} 12$ & 2 \\
\hline 23 & 74 & 1 & -3.1 & 10.9 & 7 & 4 & 3 & 5 & 6 & 3 & 8 & 0.54 & 3.5 & T6 & 2 \\
\hline 24 & 79 & 1 & -2.3 & 35.3 & 7 & 3 & 4 & 3 & 3 & 2 & 8 & 0.30 & 4.3 & T5 & 2 \\
\hline 25 & 80 & 1 & -2.7 & 38.3 & 8 & 5 & 6 & 3 & 5 & 5 & 9 & 0.52 & 6.0 & T12 & 1 \\
\hline 26 & 83 & 2 & -3.3 & 8.4 & 8 & 4 & 3 & 3 & 4 & 3 & 7 & 0.51 & 5.4 & T7 & 2 \\
\hline 27 & 90 & 2 & -2.0 & 33.3 & 7 & 3 & 3 & 3 & 4 & 4 & 6 & 0.70 & 6.5 & T10 & 3 \\
\hline
\end{tabular}

\section{Magnetic resonance imaging protocol}

Magnetic resonance examinations were performed at 1.5 T (Magnetom Essenza 1.5T, Siemens AG, Erlangen, Germany) according to standard protocols. The T1-weighted, T2-weighted, and fat-suppressed T2-weighted images were obtained in both sagittal and axial planes.

\section{Percutaneous vertebroplasty (PVP) procedure}

PVP was performed under fluoroscopic visualization (Intergris Allura Xper FD 20, Philips, Netherlands). Thirteen-gauge variable-angle sharp-tipped needles with an 11-gauge blunt trocartip cannula were used through a unilateral transpedicular (for lumbar) or parapedicular (for thoracic) approach (Fig. 1). After the needle was placed into the IVC under fluoroscopic control, the needle handle was manipulated to penetrate the sclerotic edge of the IVC to facilitate cement spreading to the surrounding bone marrow. If significant liquid accumulationin the IVC was identified by preoperative MRI, a syringe was used to draw out the liquid before the cement injection (Fig. 2).Polymethylmethacrylate (DePuy International, Leeds, UK) was used as filler. Cement injection was manipulated carefully under biplanar fluoroscopy. The IVC was filled with cement from the anterior to the posterior edge and from the left to the right side of the IVC as far as possible. The cement spreading into the surrounding bone marrow area was also identified on biplanar fluoroscopy. The injection was terminated if cement leakage was observed during the procedure. 


\section{Evaluation of outcome predictors \\ Review of medical and operative records}

Patients' medical records, including age, gender, BMI, BMD and cement volume were retrospectively reviewed. Any post-procedural complications were also recorded.

\section{Analyses of images}

All images were evaluated by consensus between two musculoskeletal radiologists, who were blind to the therapeutic effects.

\section{Evaluation of preoperative images}

As a standard protocol, all patients underwent MRI, three-dimensional reconstruction CT and anteroposterior and lateral radiographs in the standing position before PVP. Cobb's method was adapted to analyze pre-procedural curvature in all patients, based on lateral radiographs. For lumbar kyphosis, a negative value was used to facilitate post-procedural assessment of alignment correction. On preoperative MRI, the IVC was identified as an area showing marked hyperintensity (fluid collection) or signal loss (gas-containing space) on sagittal T2-weighted images (Fig. 3). Fat-suppressed T2-weighted images were utilized to depict the degrees of bone marrow edema. Picture Archiving and Communication Systems (PACS, ZLHIA, Co.Ltd, Chongqing, China) were employed to calculate the ratio of edema area over the entire vertebral body area on the lateral MRI profile. Bone marrow edema was classified into $\geq 40 \%$ or $<40 \%$ of the affected vertebral body according to the corresponding calculated ratio.

\section{Post-procedural images}

PACS was also used to calculate the ratio of the cement spreading area over the surrounding edematous area on lateral radiographs (Fig. 4). The patterns of cement spreading were classified as solid patterns (cement formed a mass in the IVC only), mild trabecular patterns (cement formed a mass in the IVC and spread into less than $50 \%$ of the edematous area on lateral radiographs), and mixed patterns (cement formed a mass and spread into more than $50 \%$ of the edematous area), according to Lane et al. [1]. Postoperative kyphosis correction was calculated using the pre-procedural Cobb's angle minus the post-procedural Cobb's angle. Based on this value, post-procedural kyphosis correction was divided into two groups. Group A had significant correction of greater or equal to 8 degrees and group B had correction of less than 8 degrees. The location of cement leakage was evaluated by postoperative CT in seven asymptomatic patients.

\section{Analyses of therapeutic outcome}

The pre- and post-procedural VAS was recorded to calculate the rate of pain relief at 7 days, 1 month, 3 months, 6 months, and 1 year after PVP. The pain improvement ratio (PIR) was calculated using the following formula:

$\mathrm{PIR}=(\mathrm{PVAS}-\mathrm{PPVAS}) / \mathrm{PVAS}$

where PVAS means pre-procedural VAS,PPVAS means post-procedural VAS.

Only if the PIR was greater than 0.6 , the clinical outcome was considered to be positive. Both the number of patients with negative results and those with positive results were recorded.

\section{Statistical analysis}

Statistical analyses (SPSS 19.0, SPSS Inc, Chicago, IL,USA) were conducted to evaluate the relationship between outcome predictors and therapeutic outcomes at all follow-up points. Pearson's Chi-squared test and Fisher's exact test were used to analyze the following factors: sex, patterns of cement distribution, degrees of bone marrow edema, and kyphosis correction ( $\geq 8^{\circ}$ or $<8^{\circ}$ ) after the procedure. Age, body mass index (BMI), bone mineral density (BMD) and cement volume were analyzed using Student's unpaired t-test. Differences were defined as statistically significant when $\mathrm{P}$ values were below 0.05 .

\section{Results}

The therapeutic effects on pain relief are shown in Table 2. The major lesion distributions were located at the thoracolumbar regions from T10 to L2 (13 out of 27), which are consistent with previous reports [1,2,7]. Of the 27 patients, 13 were male. The average age was $70.9 y e a r s$, the average BMI was21.9, the average BMD was-2.81, and the mean preoperative VAS was7.4. 
Table 2

Summary of pain relief and analyses of outcome predictors

\begin{tabular}{|c|c|c|c|c|c|c|c|c|c|c|c|c|c|c|c|}
\hline \multirow{2}{*}{$\begin{array}{l}\text { Outcome } \\
\text { parameters }\end{array}$} & \multicolumn{3}{|c|}{1 week } & \multicolumn{3}{|c|}{1 month } & \multicolumn{3}{|c|}{ 3moths } & \multicolumn{3}{|c|}{6 months } & \multicolumn{3}{|c|}{12 months } \\
\hline & INE & $E$ & $\mathrm{P}$ & INE & $E$ & $\mathrm{P}$ & INE & $E$ & $\mathrm{P}$ & INE & $E$ & $\mathrm{P}$ & INE & $E$ & $\mathrm{P}$ \\
\hline Age & 72.0 & 70.47 & 0.443 & 70.4 & 71.1 & 0.307 & 70.2 & 71.35 & 0.153 & 71.3 & 70.8 & 0.293 & 69.4 & 71.6 & 0.350 \\
\hline $\begin{array}{l}\text { Gender(male: } \\
\text { female) }\end{array}$ & $3: 5$ & $10: 9$ & 0.516 & $3: 4$ & $10: 10$ & 0.745 & $4: 6$ & $9: 8$ & 0.516 & $5: 3$ & $8: 11$ & 0.333 & $4: 4$ & $9: 10$ & 0.901 \\
\hline BMI & 21.8 & 21.1 & 0.771 & 23.0 & 20.4 & 0.659 & 17.0 & 23.5 & 0.322 & 20.4 & 21.4 & 0.306 & 23.5 & 20.1 & 0.381 \\
\hline CV & 5.20 & 4.84 & 0.403 & 5.16 & 4.88 & 0.256 & 4.79 & 5.04 & 0.691 & 5.16 & 4.86 & 0.275 & 5.11 & 4.88 & 0.527 \\
\hline BMD & -2.85 & -2.80 & 0.841 & -2.99 & -2.75 & 0.267 & -2.78 & -2.83 & 0.390 & -2.88 & -2.78 & 0.363 & -2.97 & -2.74 & 0.128 \\
\hline $\begin{array}{l}\mathrm{PE}(\geq 40 \%: \\
<40 \%)\end{array}$ & $3: 5$ & $17: 2$ & $0.037 *$ & $4: 3$ & $5: 15$ & 0.121 & $5: 5$ & $10: 7$ & 0.656 & $5: 3$ & $13: 6$ & 0.766 & $4: 4$ & $14: 5$ & 0.233 \\
\hline $\mathrm{KC}\left(\geq 8^{\circ}:<8^{\circ}\right)$ & $8: 0$ & $12: 7$ & $0.043^{\star}$ & $8: 0$ & $11: 8$ & $0.029 *$ & $10: 0$ & $9: 8$ & $0.010 *$ & 8:0 & 11:8 & $0.029 *$ & $8: 0$ & $11: 8$ & $0.029 *$ \\
\hline $\begin{array}{l}\text { CM (mass } \\
\text { type: mild } \\
\text { trabecular } \\
\text { type: mixed } \\
\text { type) }\end{array}$ & $4: 3: 1$ & $2: 9: 8$ & 0.062 & $4: 2: 1$ & $2: 10: 8$ & $0.034^{*}$ & $3: 5: 2$ & $3: 7: 9$ & 0.332 & $2: 5: 1$ & $4: 7: 8$ & 0.308 & 4:3:1 & $2: 9: 8$ & 0.062 \\
\hline
\end{tabular}

According to the preoperative protocol, the efficacy of PVP on pain due to Kummell's disease was $70.4 \%$ (19 out of 27 ) after 1 week,74.1\% after 1 month, and $63.0 \%$ (10 out of 27 ) after 3 months. Six months and one year after procedures, it remained steady at $70.4 \%$.

One patient with Kummell's disease located at T12 complained of no pain relief after PVP at all follow-up points. Moderate spondylisthesis at L2, 3 was suspected to be one cause of pain. At the last follow-up, zygapophysial joint blocks were applied bilaterally. The VAS reduced from 6 to 3 immediately after the procedure. Another patient was encountered with mild pain relief constantly after PVP. Conservative treatments including physical therapy, brace fixation and anti-osteoporosis medication failed in the pain relief. The severe positive sagittal balance of about $10 \mathrm{~cm}$ was assumed to be one of the causes of pain. Despite the insufficient pain relief, the ambulatory ability was acceptable for the patient. Further surgical treatment remains unnecessary for this patient to date.

Six patients classified into the effective group at 3 months complained of mild recurrence of pain at the 6 month follow-up. According to the protocol, two of them were classified into the ineffective group. Further anti-osteoporosis medication, physical therapies and core muscle exercises were effective in relieving pain. Five of them reported moderate pain relief at the final follow-up.

Postoperative kyphosis correction $\geq 8^{\circ}$ was related to an inferior clinical result with statistical significance at all follow-up points (Table 2). Those patients with postoperative kyphosis correction $<8^{\circ}$ reported better pain relief than did those with kyphosis correction $\geq 8^{\circ}$.

The percentage of the edematous area was a prognostic factor only at 1 week postoperatively. A preoperative edema rate $\geq 40 \%$ was significantly related to effectiveness $(P=0.037)$. After 1 month, there was no statistically-significant correlation between the area of edema and therapeutic outcomes (Table 2).

The morphology of cement was one outcome predictor only at 1 month follow-up. It seemed that mixed-type cement spreading, meaning cement permeation into more than $50 \%$ of the edematous area, had a superior pain relief effect.

Other parameters including age, sex, injected cement volume, BMD and BMI showed no statistically-significant correlation with therapeutic outcomes at any of the follow-up points.

Cement leakage was observed in seven patients (25.9\%), including two cases of intradiscal leakage, two cases of paravertebral leakage and two cases of foraminal venous leakage. All of these cement leakages were asymptomatic, which made revision surgery unnecessary.

\section{Discussion}

OVCFs have a high incidence in elderly patients $[8,10,11,13]$. In most cases, fractures heal within 4 to 6 months. However, in some cases, as a result of extreme conditions such as micro artery embolism or segmental micro-motion, OVCFs can lead to vertebral nonunion, which is known as Kummell's disease. 
Kummell's disease is a spinal disorder with a higher incidence than previously recognized. It is characterized by vertebral body avascular necrosis occurring in a delayed fashion after minor trauma [1,3,4]. It involves three phases: 1 ) back pain consequent to minor trauma, which progressively subsides, leading to an asymptomatic period; 2) recurrence of pain weeks to months after the initial insult; and 3) appearance of kyphotic spine deformities without any further trauma. All three phases must present progressively to lead to the diagnosis of Kummell's disease. A classic finding is the presence of an IVC vacuum on plain radiographs, which is more clearly visualized on CT or MRI scans.

For patients without neurological deficits, the objective is to eliminate motion at the lesion site and restore stability. Certain authors have reported that VP or KP present good clinical results for Kummell's disease without neurological symptoms[2, 3, 4]. The basic method is to stabilize the fractured vertebrae by the agglutinative effect of injected cement. Meanwhile, the cauterizing effect of the cement on the surrounding teleneuron also contributes to pain relief $[3,5,6]$.

However, Heoand Kuh[14] asserted that bone cement augmentation alone for treating Kummell's disease was insufficient because of the recurrent collapse, as well as the problems of dislodged or fragmented bone cement. They reported that the incidence of recollapse after VP was about $28.6 \%$, which could lead to subsequent symptoms. The controversial clinical results reveal the complex factors involved in treating Kummell's disease, such as preoperative imaging analysis, puncture methods, cement volume and postoperative treatments.

The purpose of this study was to report the clinical results of PVP for Kummell's disease and to comprehensively analyze possible outcome predictors. In general, the therapeutic effects of PVP for Kummell's disease are not comparable to the effects on fresh OVCFs, which are claimed to be effective in over $90 \%$ of patients $[9,10,15]$. In our study, stable therapeutic effects were achieved only in $70.4 \%$ of patients at 1 week post operation and at the final followup. The major reasons for the unsatisfactory results are complicated. Nagad et al. [15] postulated that nonunion between the cement and the bone could be the cause of spontaneous cement migration and intravertebral micro-instability, and could produce pain by stimulating the nearby disc or vertebral body. Heo and Kuh[14] asserted that refracture in the area beneath the IVC was the major reason for the recurrence of pain. Mao postulated that marrow edema in a fresh vertebral fracture was a major generator of pain [6]. The cauterization effect of cement on the intravertebral teleneuron might contribute to pain relief. The penetration of the cement into the bone trabecular space could reduce the marrow edema, and thus, contribute further to the pain relief $[10,12,15]$.

In our study, a trocar with adjustable angle tip was adopted to pierce the sclerotic edge of the IVC and facilitate cement spreading into the area beneath the edema. According to previous studies, the edematous area is a major pain generator in OVCFs, so we presume that the edematous area surrounding the IVC has a similar effect and to a large extent, contributes to the pain. Penetration of the sclerotic edge could also improve the integrity between the bone marrow and the cement to increase the micro stability.

Ultimately, eight patients achieved satisfactory cement spreading in a mixed pattern of cement distribution. Six of them showed good results at all followup points with a stable VAS of around 3 points. Two patients experienced pain aggravation in the final interview of about 4 points. Both of them responded well to conservative therapies with brace fixation. Because of the comorbidities of the two patients, including disc degeneration and segmental instability, the genuine pain mechanism was difficult to distinguish. In contrast, in two cases, the edge of the IVC was tangential to the endplate, which made it impossible to inject cement into the surrounding area. The VAS changed only from 8 to 6 in both patients, which indicated an unsatisfactory therapeutic result according to the pre-procedural protocol. At the follow-up visits, neither of them presented any significant improvement.

Despite the theoretically superior therapeutic effect of the augmentation in the edematous area, a significant difference was only established at 1 week follow-up in the analysis of the relationship between the percentage of edema and pain relief. A significant difference related to the cement morphology was only observed at 1 month follow-up. We presumed that the limited sample size might be one major reason. A well-designed, randomized, and controlled trial is required to clarify this confusion.

Several studies $[4,12,16,18]$ have demonstrated that VP can effectively improve kyphotic deformity. The therapeutic effect of kyphosis correction on pain relief remains controversial. Kim et al. asserted that overcorrection of kyphosis in patients with Kummell's disease was a negative outcome predictor [7]. In their study, kyphotic correction of over $5^{\circ}$ led to an inferior clinical result. We found a similar result in our study. Kyphotic correction of over $8^{\circ}$ was a negative predictor of pain relief at all follow-up points. We believe that too much correction of kyphosis resulted in greater preoperative instability at the fracture site. Cement augmentation might not be enough to reconstruct the instability. Overcorrection of the kyphosis could also change the relative position of the facet joints and muscle attachments, which could be one new reason for the persistent pain. Lin et al. reported that overcorrection of the pre-procedural kyphosis could increase the incidence of new symptomatic compression fractures in the adjacent vertebrae [19]. In our study, very few newonset factures were observed after PVP in the adjacent vertebrae. The influences of kyphosis correction on therapeutic outcomes require further investigation.

Limitations of this study stem from the retrospective nature of this type of analysis. The enrollment was relatively small, considering the high incidence of Kummell's disease. Being a short-term retrospective analysis, the directive meaning of this study was limited. However, considering the complicated mechanisms of low back pain in elderly patients with Kummell's disease and the fact that the main purpose of PVP is early ambulation and pain relief, we felt that the short-term therapeutic effect of PVP was more important. The VAS is the only therapeutic result parameter. No functional scores such as ODI or SF-36 were applied in our study. However, the major purpose of this type of operation is to reduce pre-procedural pain, and thus we consider that the functional score is not perfectly appropriate in a patient population with various comorbidities.

\section{Conclusion}


Percutaneous vertebroplasty for Kummell's disease was effective in only about $70.4 \%$ of patients at 1 week, $74.1 \%$ after 1 month, and $63.0 \%$ after 3 months. At one year after the procedure, the effective rate remained at $70.4 \%$. Post-procedural kyphotic correction $\geq 8^{\circ}$ was associated with poor outcomes. The percentage of edematous area over $40 \%$ and cement morphology of mixed type are positive prognostic factors for pain relief at the early stage.

\section{Declarations}

\section{Abbreviations}

PVP: Percutaneous Vertebroplasty; VAS: Visual Analogue Score; OVCF: Osteoporotic Vertebral Compression Fractures; IVC: Intravertebral Cleft; CT: Computed Tomography; MRI: Magnetic Resonance Images; PACS: Picture Archiving and Communication Systems; BMI: Body Mass Index; BMD: Bone Mineral Density

\section{Acknowledgements None.}

\section{Authors' contributions}

YBL: Data analysis, interpreting results, statistical analysis, drafting and editing manuscript. WJG: Study design, data collection, interpreting results and editing manuscript. DJH: Study design, data collection, interpreting results and editing manuscript. All authors read and approved the final manuscript.

\section{Funding}

This study is supported by the National Natural Science Research Plan in Shaanxi Province of China (No.2017JQ8056, No.2017JM8115), National Natural Science Foundation of China (No.81601898). They are used in the data collection, analysis and writing the manuscript.

\section{Availability of data and materials}

The datasets used and analyzed during the current study are available from the corresponding author on reasonable request.

\section{Ethics approval and consent to participate:}

This article does not contain any studies with human or animals rights violation.

Consent for publication: Not applicable.

Competing interests: The authors declare that they have no competing interests.

\section{References}

1.

Lane Jl, Maus TP, Wald JT, Thielen KR, Bobra S, Luetmer PH. Intravertebral clefts opacified during vertebroplasty: pathogenesis, technical implications, and prognostic significance. Ajnr Am J Neuroradiol. 2002;23(10):1642-6.

2.

Yang H, Gan MJ, Mei X, Shen X, Wang G, Chen L. Kyphoplasty for the treatment of Kummell's disease. Orthopedics. 2010;33(7):479. 3.

Peh WC, Gelbart MS, Gilula LA, Peck DD. Percutaneous vertebroplasty: treatment of painful vertebral compression fractures with intraosseous vacuum phenomena. AJR Am J Roentgenol. 2003;180(5):1411-7.

4.

Wiggins MC, Sehizadeh M, Pilgram TK, Gilula LA. Importance of intravertebral fracture clefts in vertebroplasty outcome. AJR Am J Roentgenol. 2007;188(3):634-40.

5.

Ha KY, Lee JS, Kim KW, Chon JS. Percutaneous vertebroplasty for vertebral compression fractures with and without intravertebral clefts. J Bone Joint Surg Br. 2006;88(5):629-33.

6.

Mao H, Zou J, Geng D. Osteoporotic vertebral fractures without compression: key factors of diagnosis and initial outcome of treatment with cement augmentation. Neuroradiology. 2012;54(10):1137-43.

7.

Kim YJ, Lee JW, Kim KJ, Chung SK, Kim HJ, Park JM. Percutaneous vertebroplasty for intravertebral cleft: analysis of therapeutic effects and outcome predictors. Skeletal Radiol. 2010;39(8):757-66.

8.

Gerling MC, Eubanks JD, Patel R, Whang PG, Bohlman HH, Ahn NU. Cement augmentation of refractory osteoporotic vertebral compression fractures: survivorship analysis. Spine. 2011;36(19):1266-9.

9. 
Li B. Percutaneous vertebroplasty for acute severe osteoporotic vertebral compression fractures with high viscosity bone cement. Laser J.

2013;34(6):118-9.

10.

Zhang D, Luo Z. Analysis of the clinical outcomes of high-and low-viscosity bone cement in the treatment of osteoporotic vertebral compression fractures by percutaneous vertebral angioplasty(PVP). Med Sci J Cent South China. 2016;23(5):48-51.

11.

Ye LQ, Liang, Jiang XB, Yao ZS, Lu H, Qiu T, et al. Risk factors for the occurrence of insufficient cement distribution in the fractured area after percutaneous vertebroplasty in osteoporotic vertebral compression fractures. Pain Physician. 2018;21(1):E33.

12.

En X, Hao DJ. Percutaneous kyphoplasty versus conservative treatment in acute and subacute osteoporotic vertebral compression fractures (OVCF): a double-blinded, randomized controlled clinical trial (RCT) in the population of Western China. Spine J. 2013;13(9):90-1.

13.

Hadjipavlou AG, Tzermiadianos MN, Katonis PG. Percutaneous vertebroplasty and balloon kyphoplasty for the treatment of osteoporotic vertebral compression fractures and osteolytic tumours. J Bone Joint Surg Br. 2005;87(12):1595.

14.

Heo DH, Kuh SU. Progressive, repeated lumbar compression fracture at the same level after vertebral kyphoplasty with calcium phosphate cement. Case report. J Neurosurg Spine. 2007;6(6):559.

15.

Nagad P, Rawall S, Kundnani V. Postvertebroplasty instability: report of 5 cases. J Neurosurg Spine. 2012;16(4):387-93.

16.

Hu YC, Hart DJ. Complications of vertebroplasty and kyphoplasty. Tech Reg Anesth Pain Manag. 2007;11(3):164-70.

17.

Hierholzer J, Fuchs H, Westphalen K, Baumann C, Slotosch C, Schulz R. Incidence of symptomatic vertebral fractures in patients after percutaneous vertebroplasty. Cardiovasc Intervent Radiol. 2008;31(6):1178-83.

18.

Chang WS, Lee SH, Choi WG. Unipedicular vertebroplasty for osteoporotic compression fracture using an individualized needle insertion angle. Clin J Pain. 2008;23(9):767-73.

19.

Lin WC, Cheng TT, Lee YC, Wang TN, Cheng YF, Lui CC. New vertebral osteoporotic compression fractures after percutaneous vertebroplasty: retrospective analysis of risk factors. J Vasc Interv Radiol. 2008;19(2 Pt 1):225-31.

\section{Figures}




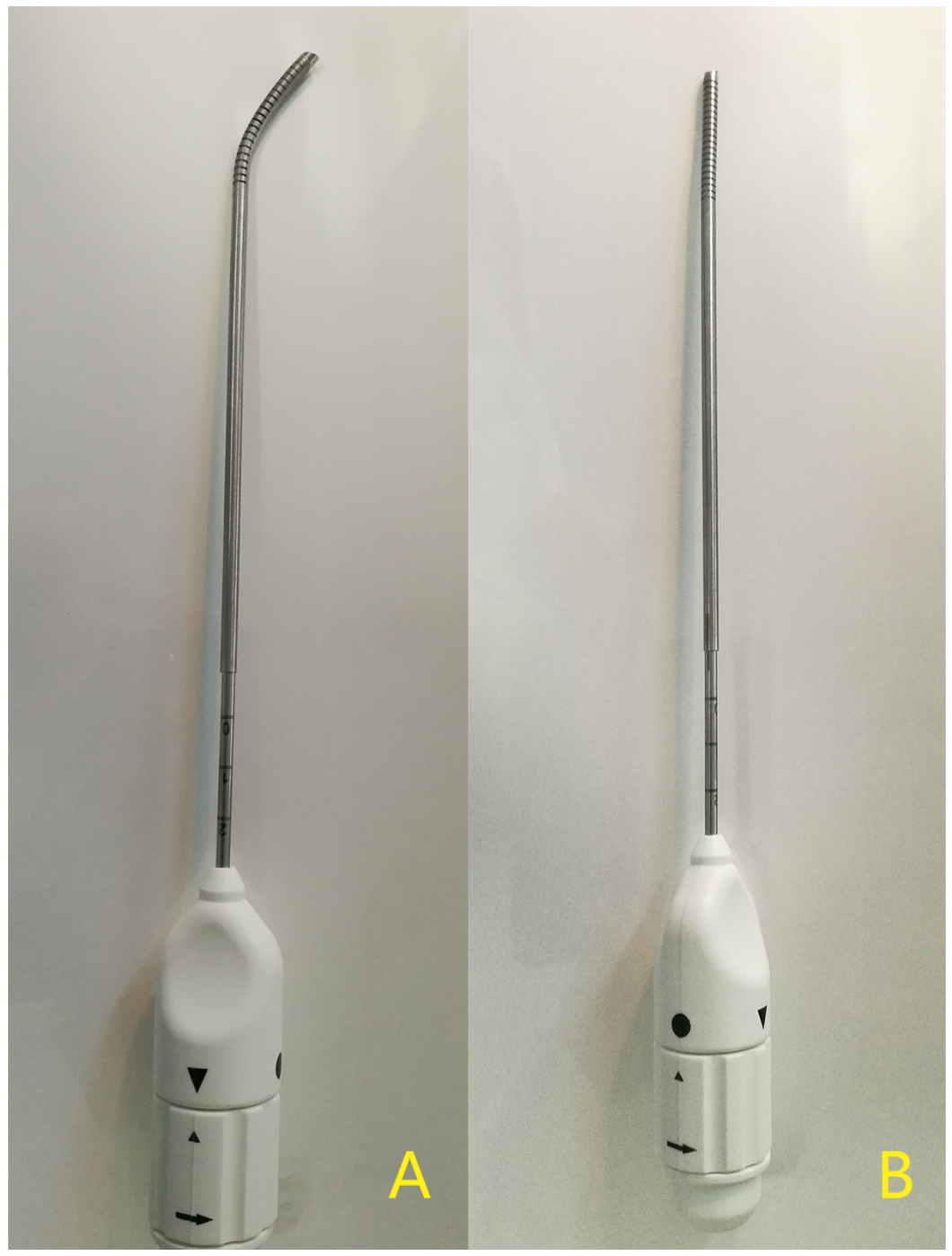

Figure 1

A variable-angle sharp-tipped needle was used to penetrate the sclerotic edge of the lesion area in the vertebrae to facilitate spreading of the cement. 


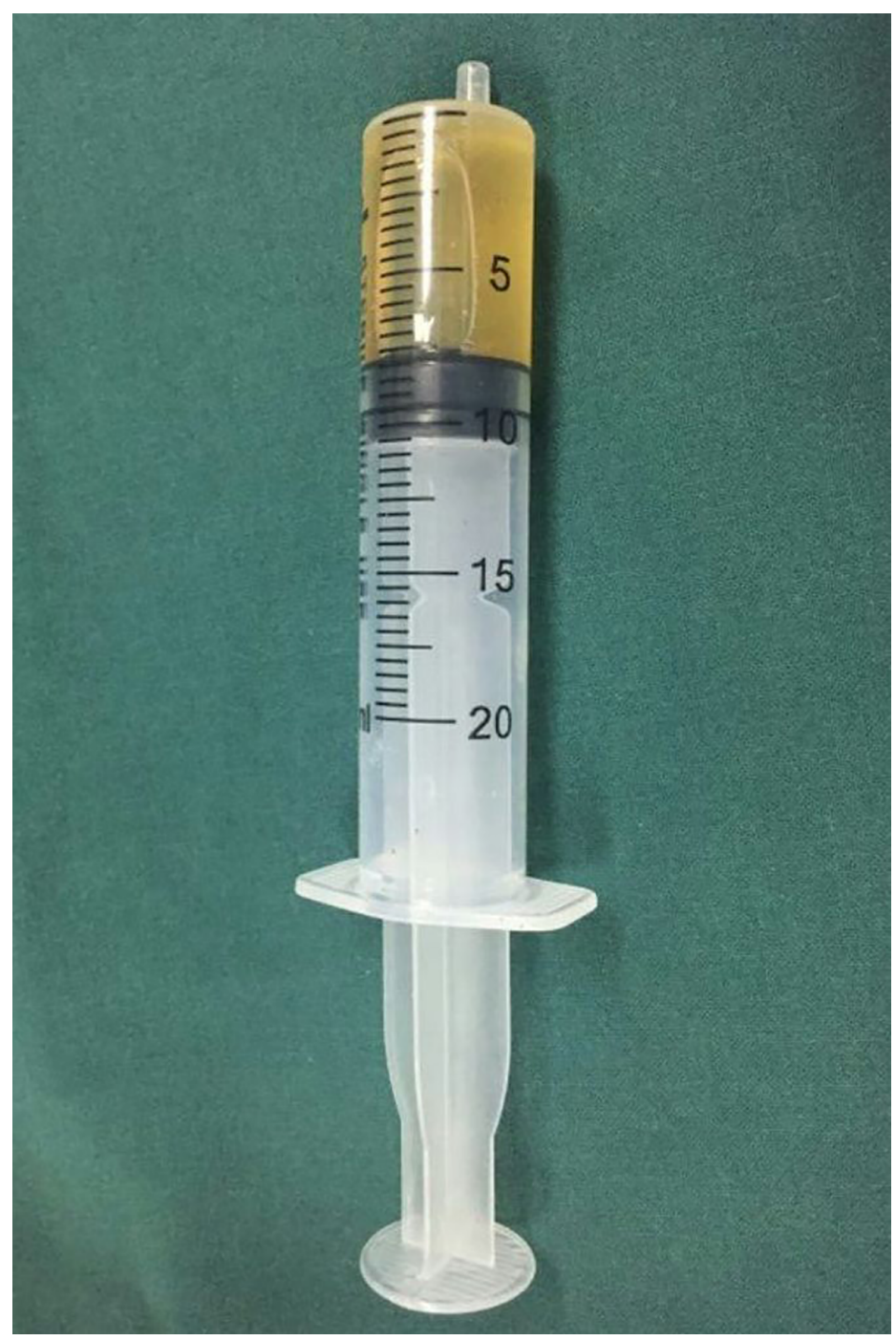

Figure 2

Synovial fluid was removed from the lesion before cement injection. 


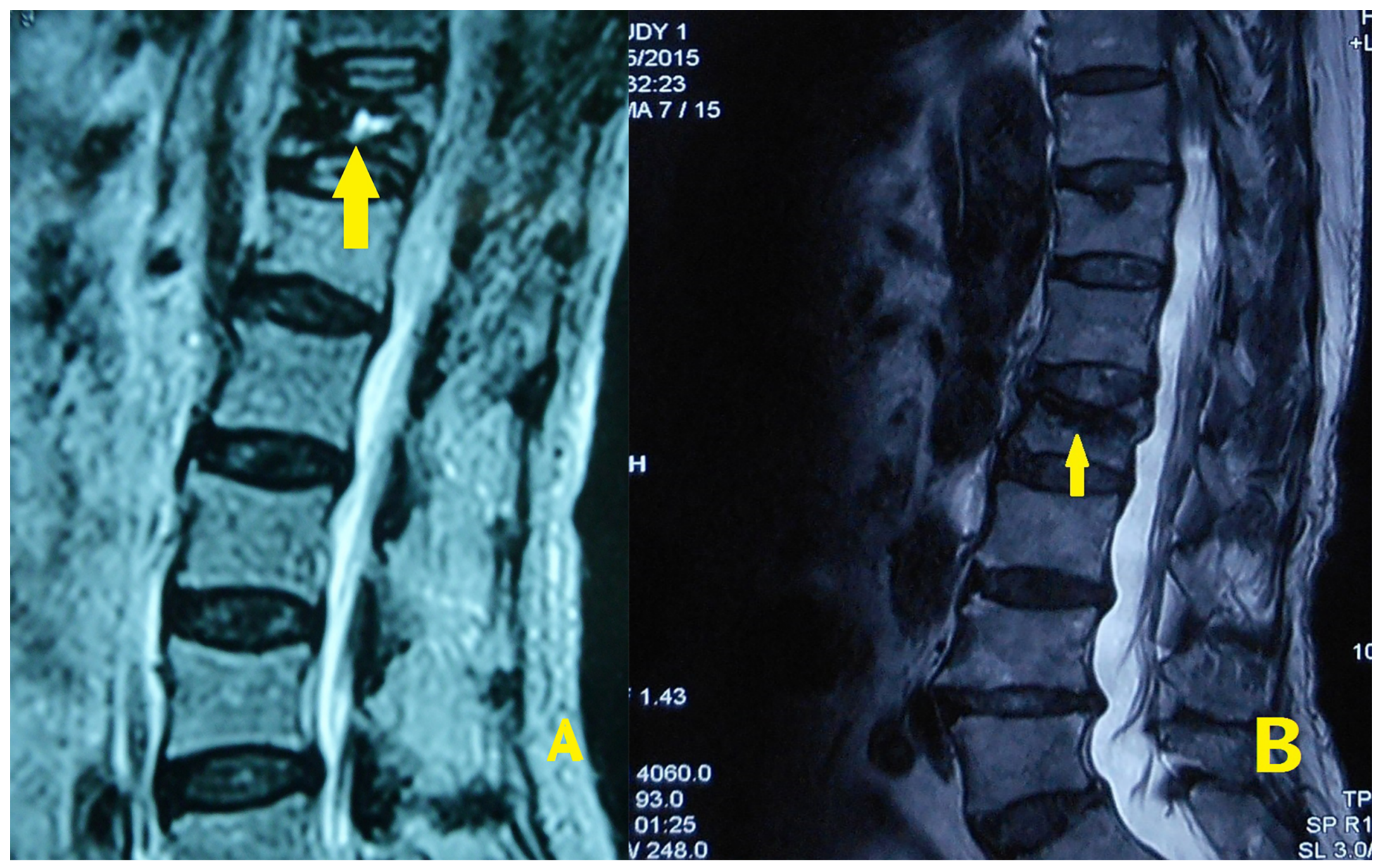

Figure 3

Typical MRI features of Kummell's disease in T2-weighted images, fluid collection (A) or gas-containing space (B)

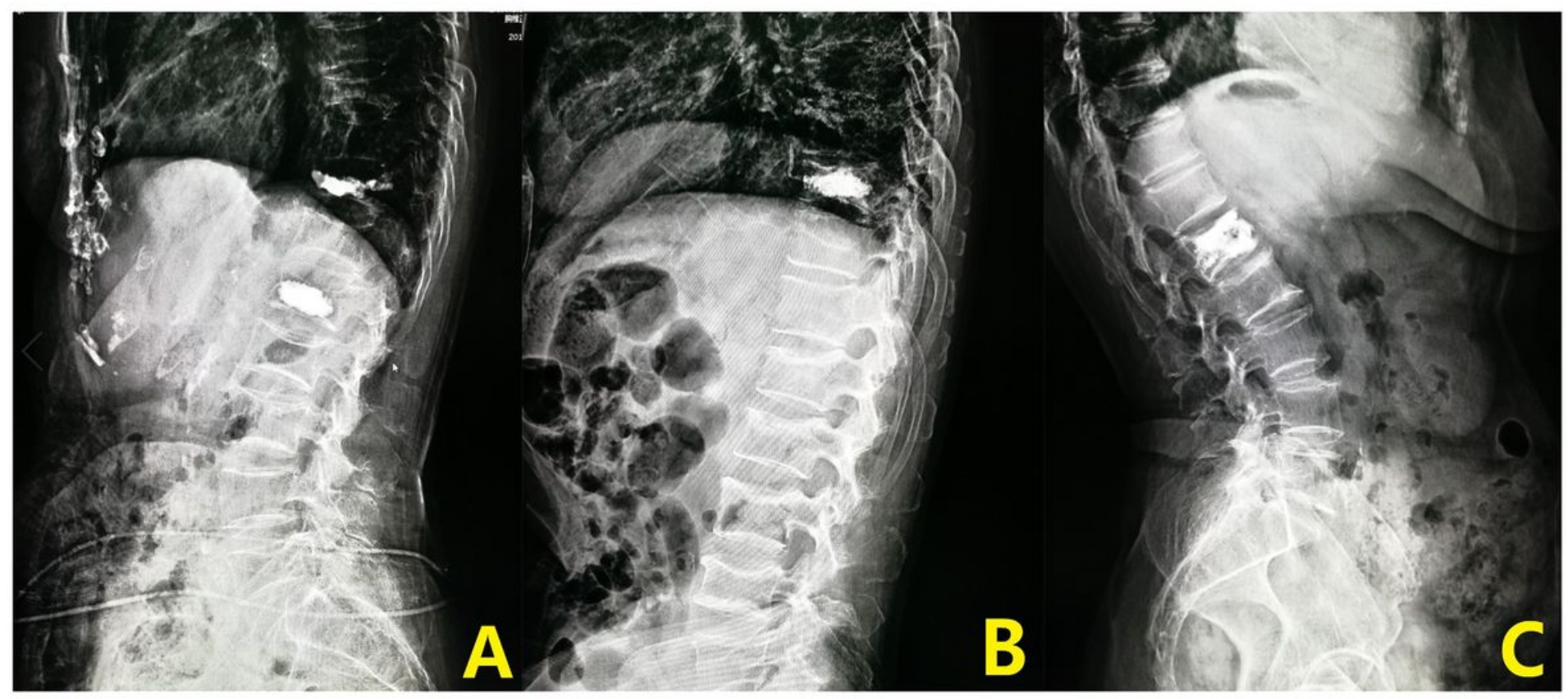

\section{Figure 4}

Various types of cement distribution after cement injection were identified using PACS based on lateral radiographic views. According to the ratio of the cement area over the edematous area on lateral view, the cement morphologies were divided into mass type (A), trabecular type (B) and mixed type (C). 